\title{
Spiking Chemical Sensor (SCS): A new platform for neuro-chemical sensing
}

\author{
Pantelis Georgiou*, Iasonas F. Triantis ${ }^{\dagger}$, Timothy G. Constandinou ${ }^{\dagger}$ and Chris Toumazou ${ }^{\dagger}$ \\ ${ }^{\dagger}$ Institute of Biomedical Engineering, *Department of Electrical and Electronic Engineering \\ Imperial College London SW7 2AZ, UK \\ Email:\{pg200, i.triantis, t.constandinou, c.toumazou\}@imperial.ac.uk
}

\begin{abstract}
A spiking chemical sensor (SCS) is presented for detection of neurogenic ion concentration associated with active nerve fibres in nerve bundles. Based on the "integrate-and-fire" circuit, the SCS uses a chemically-modified ISFET front end encoding the sense data in the spike domain. Used in an array, it provides a spatio-temporal map of chemical activity around the nerve bundle which may be relayed off chip using low power asynchronous communication hardware. The circuit is shown to be tunable to yield a linear relation with either $\mathrm{pH}$ or actual hydrogen ion concentration. Furthermore, its compact pixel footprint in addition to efficient use of the sensing surface, makes it ideal for use in neuro-chemical imaging.
\end{abstract}

\section{INTRODUCTION}

Devices for monitoring neural activity can be used for a range of applications including studies of neurophysiology and neuropathology, diagnostics, drug monitoring and rehabilitation [1]. Although nerve conduction is based on ionic current flow, most neural monitoring studies focus on bioelectric recording (recording of electroneurogram or ENG) with very few studies using ionic sensing in-vitro or in-vivo. This is almost entirely due to the conventional apparatus for ion sensing in chemical laboratories being cumbersome, slow and innapropriate for small concentrations [2].

ISFET (Ion Sensitive Field Effect Transistor) based chemical sensors, first introduced by Bergveld [2], have gained considerable interest due to their ion sensitivity, fast temporal response, compact size and prospect of monolithic integration [3]. Although much of the initial literature predicted ISFETs to be mainly used as future tools for electrophysiological measurements, the sensors were not further developed for biomedical applications in particular, but rather for ion sensing in general [2].

In order to develop an ISFET-based interface for measuring neurogenic ion concentration variations [4], certain customization is needed. The main requirements of a neurochemical interface include robustness, compactness, spatiotemporal selectivity and ionic specificity. To achieve this, custom designed ISFETs based on CMOS technology are integrated with compact local processing to realise an "intelligent chemical sensor". This can be combined with a multitude of such sensors in an array, providing spatio-temporal sensing and the added advantage of sensor failure immunity (by redundancy). The next step is to characterise the sensors by $\mathrm{pH}$ measurements which can then be adjusted to be selective to specific ions that relate to neural conduction. Although ISFETs are intrinsically sensitive to hydrogen ions, the selectivity may be changed by depositing various ionophores on the surface [5].

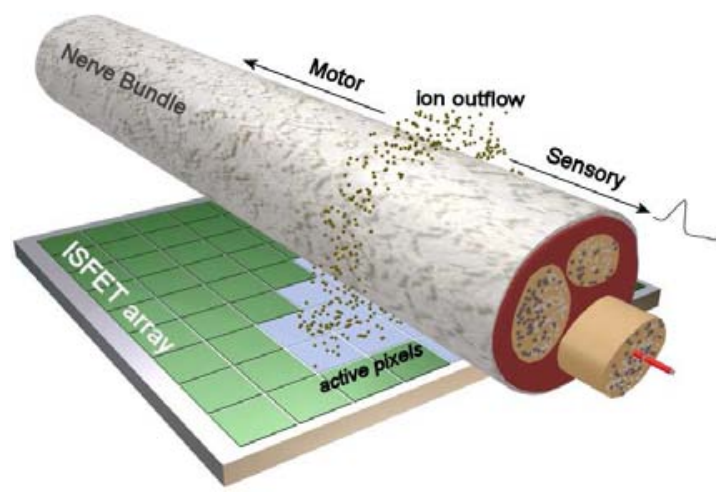

Fig. 1. Potassium ion sensing from a nerve bundle. The ISFET array is used to generate a spatio-temporal map of chemcial activity.

Presented here is an integrated chemical image sensor with local signal conditioning and pre-processing circuitry. Fabricated in a commercially available CMOS technology, the SCS includes an ISFET-based device modified for potassium ion sensitivity in addition to bias, drive and signal conversion circuitry. Moreover, a matrix of sensors has been tesselated on a single chip, forming the basis of a chemical image sensor and additionally improving robustness and accuracy through redundancy. Furthermore, by embedding additional local processing, added functionality can be realised in chemical sense applications, such as spatiotemporal chemical filtering, multichemical sensing and action potential detection from neural ensembles.

In neural sensing systems a significant portion of power dissipation and chip area is attributed to the ADC and the digital signal processing. In order to overcome this issue, a neuromimetic circuit has been adopted to perform the processing of the recorded bio-signals and convert them to a form that is compatible an external device. The processing circuitry adopted within this sensor is a low power "integrate-and-fire" neuron circuit, (I\&F), proposed by Indiveri [6], encoding the sensor information in the spike domain with multiple sensor fusion and off-chip transmission facilitated using a standard AER (Address Event Representation) [7] protocol. 
This paper is organised as follows. First, one of the target applications is explained, specifically spatio-temporal selectivity in nerve bundle interfacing, which was the motivation for making an array of spiking chemical sensors. The advantages of chemical neural sensing and the circuit used are also specified. This is followed by the circuit implementation in weak inversion CMOS and explanation of how sensor linearity is achieved for both $\mathrm{pH}$ and hydrogen ion concentration. Implementation of the ISFET sensor in standard CMOS technology is also explained followed by a discussion and concluding remarks.

\section{THEORY}

\section{A. Neural Conduction}

Based on nerve conduction theory, the membrane of a "firing" neuron exchanges ions with the surrounding extracellular space, through so called ion-pumps. The firing of a group of nerves in one or more fascicles (a group of nerves) creates ionic currents that flow between the fascicles to the extracellular space [8], which generate detectable ENG signals. Thus, localised ionic concentration variations taking place during an action potential occurrence can be detected using modified ISFETs (i.e chemFETs), tailored for specific ion selectivity, close to the active nodes of Ranvier, Fig. 1. The potential benefits of neurochemical sensing using arrays versus conventional bioelectric recording methods include increased myoelectric interference immunity, fascicle-specific monitoring, and distinction between sensory and motor signals when both take place in a nerve bundle [4].

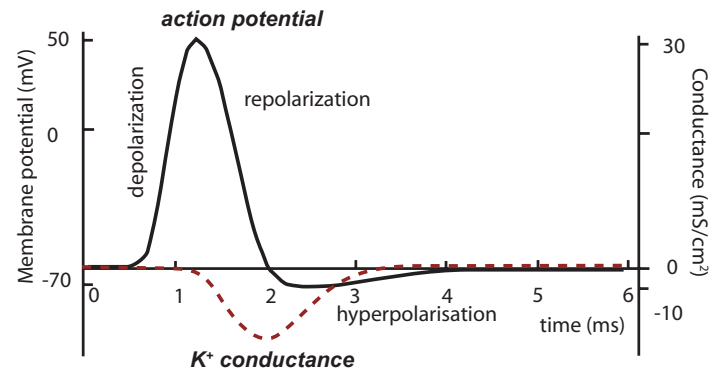

Fig. 2. Change in conductance in sodium and potassium when an action potential occurs [9]

\section{B. Ion Sensing}

Considering there is an outflow of potassium ions for each action potential, having an array of chemFETs in close proximity with the nerve bundles allows for detection in variation of potassium concentration as the action potential propagates. Fig. 2 depicts the measured change in ionic conductance when potassium and sodium ions are flowing in and out of the neuron during an action potential. Chemical spatiotemporal mapping of these events along the nerve allows both identification of signal direction, but also neuron diameters by the speed of propagation. The first allows for discrimination between motor or sensory signals and the latter allows for signal association with different categories of organs or muscle groups.

Development of chemFETs by ISFET modification has been reported in [10]. Fig. 3 shows calibration curves conducted using potassium chemFETs from IMT, Neuchatel Switzerland. The results demonstrate the appropriateness of ISFET based potassium sensors for sensing concentrations well within the range observed during neural conduction (typically $5 \mathrm{mM}$ $150 \mathrm{mM}[8])$.

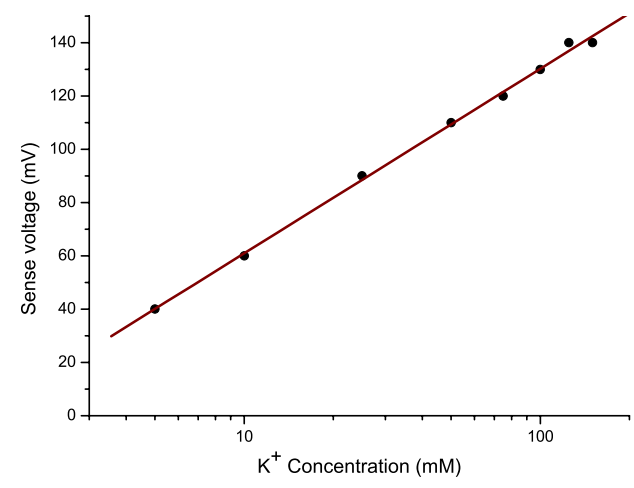

Fig. 3. Potassium chemFET calibration curve.

\section{Integrate and Fire}

Integrate and fire as its name suggests integrates a sensory current on a capacitive node creating a spike or digital pulse after a threshold has been reached resetting the capacitor. As a result sensor information, in this case concetration of ions, is conveyed in the frequency domain (spike rate), i.e. pulse position modulation (PPM).

Encoding the sensor data in the spike domain has the advantages of improved versatility in dynamic range (i.e. tradability between dynamic range and response time), robustness in signal integrity, in addition to inherent compatibility to addressevent encoding. AER is an established, asynchronous method of relaying single-bit data from multiple pixels by transmitting address-events i.e. the pixel co-ordinates of the active pixel. This reduces bandwidth and therefore also power, as it is an event-driven (data-dependant) architecture, in addition to being easily scalable for sparse data sets [7]. Relaying the chemical information in this fashion using an array of SCSs allows for energy efficient spatio-temporal mapping of potassium variations across the nerve bundles for the applications mentioned.

\section{CiRcuit Description}

Fig. 4 shows the schematic of the SCS (excluding the AER handshake switches). The circuit comprises of a custom ISFET device, the H-Cell module [11] and a leaky integrate and fire neuron [6]. The ISFET device sinks an output current which is dependant on the $\mathrm{pH}$ of the solution. This is due to the binding of hydrogen ions to the silicon nitride surface (CMOS passivation layer) above the gate forming a capacitive coupling with the $\mathrm{Ag} / \mathrm{AgCl}$ reference electrode [2]. The ISFET is biased in the weak inversion region of operation to minimise power 


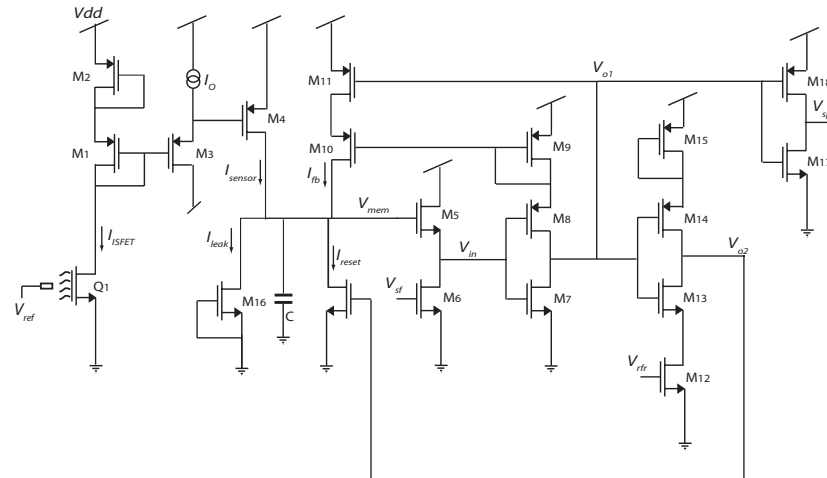

Fig. 4. Circuit schematic of chemical pixel sensor. The circuit comprises of the H-cell with the ISFET sensor and a leaky integrate and fire neuron circuit.

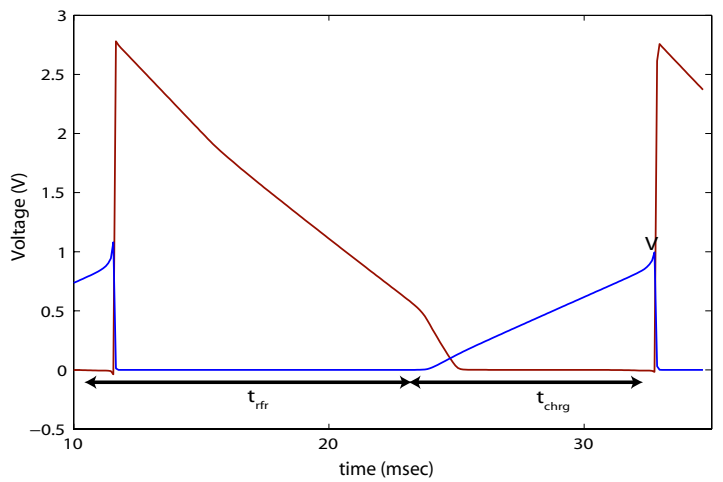

Fig. 5. Spike generation period set by charging and discharging of capacitive nodes.

consumption and exploit the exponential device characteristic. The output current relation to hydrogen ion concentration is given by Eqn. 1 and to $\mathrm{pH}$ by Eqn. 3, where $K_{\text {chem }}$ is a $\mathrm{pH}$ independent constant, $n$ the sub-threshold slope factor, $U_{t}$ is the thermal voltage and $a$ is a dimensionless sensitivity parameter relating the ISFET's sensitivity to the Nernstian relationship. The function of the $\mathrm{H}$-cell is to linearise the drain current to hydrogen ion concentration relationship, as analysed in [11]. This sensor current is fed directly into the leaky I\&F circuit, where the current magnitude representation is translated into the frequency domain (spike rate).

$$
\begin{gathered}
I_{I S F E T}=I_{0} e^{\frac{V_{r e f}}{n U_{t}}} K_{\text {chem }}\left[H^{+}\right]^{\frac{a}{n}} \\
K_{\text {chem }}=e^{\frac{-\gamma}{n U_{t}}} \\
I_{I S F E T}=I_{0} e^{\frac{V_{r e f}}{n U_{t}}} K_{\text {chem }} e^{\frac{2.3 a p H}{n}}
\end{gathered}
$$

The spike-rate dependence on the sense current is expressed in Eqn. 4, where $I_{\text {sensor }}$ is the linearised sense-current output (from the H-Cell) and is assumed to be considerably larger than the leakage current $I_{\text {leak }}$. The maximum frequency, $f_{r f r}=1 / t_{r f r}$, is determined by the time taken for the charge on node $V_{02}$ to discharge through device M12, allowing for the capacitor to start charging up again and spike shown in Fig. 5. This time is tunable by adjusting voltage $V_{r f r} . C$ is the integrating capacitor and $V$ is the voltage at which the neuron spikes, i.e. the threshold level at time $t_{\text {chrg }}=$ $C . V / I_{\text {sensor. }}$. What can be observed from this equation is that when $I_{\text {sensor }}<<C$.V. $f_{r f r}$ then $f_{\text {spike }}$ increases linearly (with sense current), but as the sense current continues to increase then $f_{\text {spike }}$ converges to the maximum spiking rate (i.e. $\left.f_{r f r}\right)$. Thus, operating the circuit in the linear region of operation (where the hydrogen ion concentration is proportional to the sense current), gives a linear relation with spike rate when the current range is smaller than C.V. $f_{r f r}$. When operating at current ranges which make the frequency plateau at $f_{r f r}$ the current to spike-frequency relation is compressive. Considering that the sense current to $\mathrm{pH}$ relation given by Eqn. 3 , is exponential, operating with similar input currents in this region also yield a linear relationship with $\mathrm{pH}$.

$$
f_{\text {spike }}=\frac{I_{\text {sensor }} \cdot f_{r f r}}{I_{\text {sensor }}+C \cdot V \cdot f_{r f r}}
$$

The feedback current $I_{f b}$ serves to limit the short-circuit currents in the inverters during switching and therefore reducing the power consumption. Furthermore, the voltage bias $V_{s f}$ defines the neurons threshold voltage [6].

\section{Simulated RESUlTS}

This circuit was simulated using the Spectre simulator (v5.1.41usr2) under the Cadence IC design environment with foundry supplied models for a commercial $0.35 \mu \mathrm{m}$ CMOS process. The ISFET was implemented using a spice behavioral macro-model derived from fabricated devices. The circuit bias conditions used are as follows: $V_{r e f}=874 \mathrm{mV}, V_{s f}=V_{r f r}=400$ $\mathrm{mV}$ and $I_{0}=100 \mathrm{nA}$. These were chosen assuming $\gamma=324 \mathrm{mV}$, giving an ISFET current of $11.6 \mathrm{nA}$ for $\mathrm{pH}$ 7. The spike frequency dependence on hydrogen ion concentration is shown in Fig. 6.b, with frequency increasing linearly with the ISFET sense current and Fig. 6.a shows spike trains produced for 3 different $\mathrm{pH}$ values. Fig. 6.c shows the spike frequency dependence on the $\mathrm{pH}$ of the test solution, during operation in the "compressive region", yielding a linear relationship. The peak power consumption at the maximum spike frequency of operation was $35 \mu \mathrm{W}$ at $3.3 \mathrm{~V}$ supply voltage.

\section{ISFET FABRICATION}

The ISFET sensors are based on standard MOSFET structures with the polysilicon gate being extended to create the sensing surface of charge, mainly hydrogen ions, binding to the silicon nitride passivation layer. The methodology used to design the ISFETs is adopted by Hammond et al [3] where by the polysilicon gate is connected via the metal layers to the top metal layer covered by silicon nitride passivation. This is left floating to be biased by an external reference electrode.

The layout of the SCS is shown in Fig 7 with the device cross section shown on the bottom. The ISFET sensor is designed with a large gate area to minimise $1 / \mathrm{f}$ noise. The 

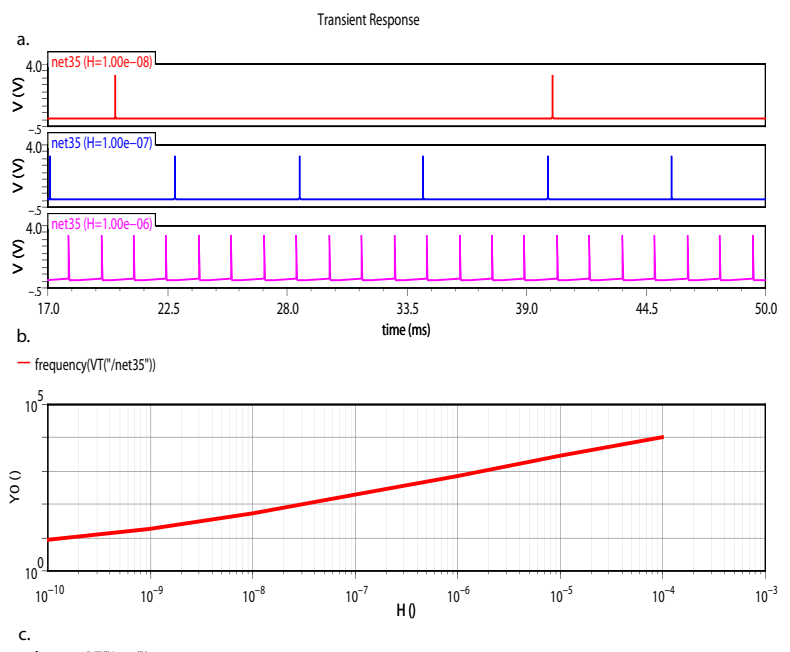

C.

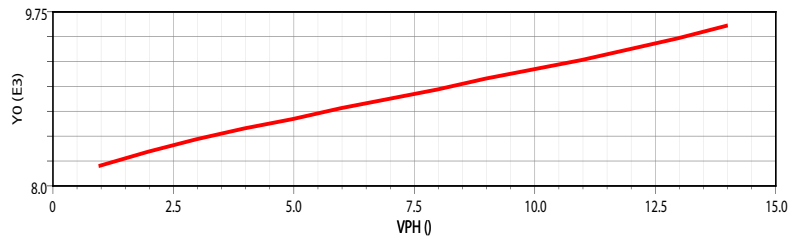

Fig. 6. a.) Spike generation for $\mathrm{pH} \mathrm{6,7}$ and 8. b.) Spike frequency response (linear) with the hydrogen ion concentration of test solution. c.) Spike frequency response (linear) with the $\mathrm{pH}$ of the test solution.

total pixel size with the I\&F circuitry is $65 \mu \mathrm{m} \times 100 \mu \mathrm{m}$. Implementing the ISFET in CMOS enables the active gate to be extended to the top metal layer. Furthermore, this is designed to cover the entire pixel area, thus achieving a near $100 \%$ effective fill factor (active sensor area to pixel ratio).

The ISFET is then modified for potassium detection by deposition of a potassium ionophore. A photocurable polymer ion-selective membrane based on urethane acrylate with good adhesion to the sensor surface is applied for direct monitoring of the potassium ion concentration.

\section{CONCLUSION}

This paper describes the front-end implementation of an ISFET-based chemical sensor with embedded neuromimetic signal processing. An array of spiking chemical sensors has been designed for monitoring of neural signatures. This was developed to provide two advanced modes of selectivity; directionality and nerve fibre distinction by diameter. The ISFET has been custom built so that it makes $100 \%$ use of the pixel surface. The I\&F stage has been designed for minimimal power and area. The spike rate has been shown to be tunable to be either linear with hydrogen ion concentration or with $\mathrm{pH}$.

\section{ACKNOWLEDGMENT}

The authors would like to acknowledge Amir Eftekhar and Li Xiaoying for their contributions, Dr. Julius Georgiou of the

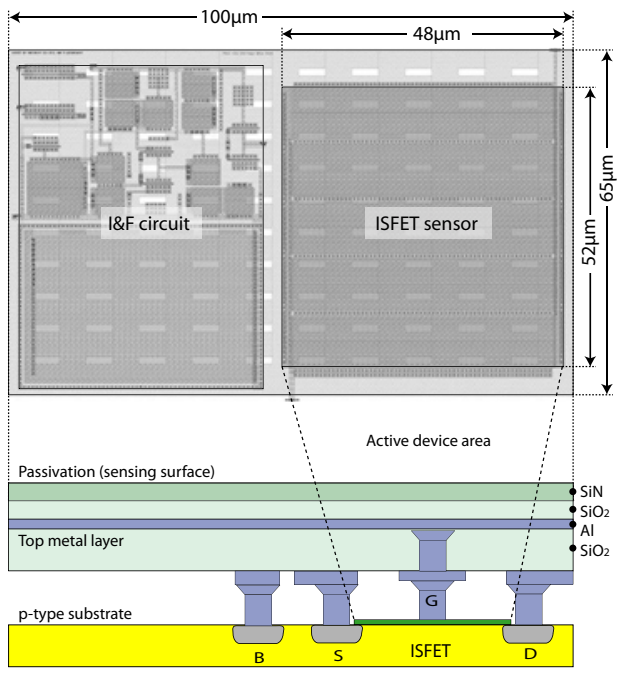

Fig. 7. Layout of the chemical pixel.

University of Cyprus for providing access to fabricating silicon and Dr. Peter van der Wal of IMT Neuchtel, Switzerland, for their potassium chemFET test prototypes.

\section{REFERENCES}

[1] I. Triantis, An Adaptive Amplifier for Cuff Imbalance Correction and Interference Reduction in Nerve Signal Recording. $\mathrm{PhD}$ thesis, University College London, London, 2005.

[2] P. Bergveld, "Thirty years of isfetology - what happened in the past 30 years and what may happen in the next 30 years," Sensors and Actuators B-Chemical, vol. 88, no. 1, pp. 1-20, 2003.

[3] P. A. Hammond, D. Ali, and D. R. S. Cumming, "Design of a single-chip ph sensor using a conventional $0.6-\mathrm{mu} \mathrm{m}$ cmos process," Ieee Sensors Journal, vol. 4, no. 6, pp. 706-712, 2004.

[4] I. Triantis and C.Toumazou, "Advanced mixed-mode nerve cuff interface." GB Patent Number 0613698.0 - patent pending, 2006.

[5] A. Bratov, N. Abramova, C. Dominguez, and A. Baldi, "Ion-selective field effect transistor (isfet)-based calcium ion sensor with photocured polyurethane membrane suitable for ionised calcium determination in milk," Analytica Chimica Acta, vol. 408, no. 1-2, pp. 57-64, 2000.

[6] G. Indiveri, "A low-power adaptive integrate-and-fire neuron circuit," in Circuits and Systems, 2003. ISCAS '03. Proceedings of the 2003 International Symposium on, vol. 4, pp. IV-820, 2003.

[7] M. Mahowald, VLSI Analogs of Neuronal Visual Processing: A Synthesis of Form and Function. PhD thesis, Caltech Pasadena, California, 1992.

[8] J. Malmivuo and R. Plonsey, Bioelectromagnetism: Principles and Applications of Bioelectric and Biomagnetic Fields. Oxford University Press, 1995.

[9] A. L. Hodgkin and A. F. Huxley, "A quantitative description of membrane current and its application to conduction and excitation in nerve," Journal of Physiology, vol. 117, pp. 500-554, 1952.

[10] E. A. Moschou and N. A. Chaniotakis, "Potassium selective chemfet based on an ion-partitioning membrane," Analytica Chimica Acta, vol. 445 , no. 2 , pp. 183-190, 2001.

[11] L. Shepherd and C. Toumazou, "Weak inversion isfets for ultra-low power biochemical sensing and real-time analysis," Sensors and Actuators B-Chemical, vol. 107, no. 1, pp. 468-473, 2005. 